\title{
Um Jogo Móvel Baseado em Localização para Motivar e Acompanhar Estudantes no Processo de Ensino-Aprendizagem
}

\author{
Luis Eduardo Costa Laurindo - Faculdade Estácio | CEUT \\ luiseduardocosta417@gmail.com \\ Ivan Rodrigues de Moura - Universidade Federal do Maranhão \\ ivan.rodrigues@Isdi.ufma.br \\ Matias Romário Pinheiro dos Santos - Universidade Federal da Bahia \\ matiasrps@ufba.br
}

Resumo. Diante do atual contexto educacional, percebe-se dificuldade em motivar e envolver os estudantes nas atividades escolares por meio de métodos tradicionais de ensino. Pesquisadores acreditam que esta insatisfação pode ser causada pelo fato de que os jovens que convivem com mídias digitais em seu dia a dia tenham dificuldades para aprender por meio dos métodos tradicionais. Assim, este artigo desenvolveu um Jogo Móvel Baseado em Localização (JMBL) com recursos de Realidade Aumentada (RA). O objetivo é proporcionar aos estudantes um ambiente lúdico e motivador para atividades escolares. Por meio de uma avaliação ergonômico-pedagógica, os resultados demonstraram que o JMBL projetado permite uma experiência agradável ao usuário e possui uma estrutura pedagógica capaz de contribuir significativamente para a aprendizagem do estudante.

Palavras-chaves: jogo móvel baseado em localização, realidade aumentada, engajamento, ensino-aprendizagem.

\section{A Location-Based Mobile Game to Motivate and Accompany Students in the Teaching-Learning Process}

\begin{abstract}
Given the current educational context, is perceived difficulty in motivating and involving students in school activities through traditional teaching methods. Researchers believe that the dissatisfaction may be caused by the fact that young people who live with digital media in their daily lives have difficulty learning through traditional methods.Thus, this article developed a gamified Location-Based Mobile Game gamed with augmented reality features. The aim is to provide students with a playful and motivating environment for school activities. Through an ergonomicpedagogical evaluation, the results demonstrated that the JMBL designed allows a pleasant user experience and has a pedagogical structure capable of contributing significantly to student learning.
\end{abstract}

Keywords: location-based mobile game, augmented reality, virtual environment, teaching-learning.

\section{Introdução}

Os métodos tradicionais de ensino apresentam dificuldades em manter a maioria dos estudantes motivados e engajados em sala de aula (FERNANDES e RIBEIRO, 2018). Os autores acreditam que esta insatisfação pode ser causada pelo fato de que os 
jovens que convivem com mídias digitais em seu dia a dia tenham dificuldades para aprender por meio dos métodos tradicionais. Ainda segundo eles, vários pesquisadores acreditam no potencial do uso de tecnologias para engajar e motivar estudantes no processo de aprendizagem, com intuito de torná-lo mais atrativo e dinâmico.

Os jogos são exemplos de mídias digitas que tem o potencial de proporcionar aos usuários uma experiência prazerosa e divertida. O emprego de jogos como estratégia didática surgem como alternativa ao modelo tradicional de ensino, com potencial de oportunizar aos estudantes uma aprendizagem de forma lúdica, com intuito de encorajar, engajar, motivar, envolver e entreter o estudante em atividades escolares (UZUNCA e JANSEN, 2016).

Assim os JMBLs surgem como uma alternativa inovadora de ensino devido suas características de não aprisionar o jogador a tela de um console, pois suas atividades lúdicas acontecem no espaço urbano, com finalidade de incentivar a realização de atividades em grupos ao ar livre e atividades físicas (PRITAMI e MUHIMMAH, 2018) e (VASSILAKIS et al. 2017). Os JMBLs são uma subclasse dos jogos pervasivos, no qual utilizam o ambiente virtual e o espaço urbano para fins de jogos, por meio de tecnologias digitais móveis e sistemas de geolocalização (LEMOS, 2010). As ações executadas pelos jogadores no espaço urbano, influenciam diretamente nos resultados do ambiente virtual. A tecnologia de RA pode ser integrada aos JMBLs com intenção de potencializar suas atividades.

Diante das deficiências elencadas, este artigo objetiva apresentar um JMBL integrado com a tecnologia de RA com objetos em 2D, com propósito de fornecer um ambiente lúdico e engajador, para motivar os estudantes na realização de atividades escolares. O JMBL disponibiliza para o professor uma aplicação web para criar e gerenciar o conteúdo didático disponibilizado para os estudantes e acompanhar o desempenho dos mesmos.

Este artigo está organizado da seguinte forma: a Seção 2 apresenta a metodologia utilizada para o desenvolvimento deste artigo; a Seção 3 expõe os conceitos sobre JMBLs; a Seção 4 apresenta os trabalhos relacionados; a Seção 5 apresenta o JMBL concebido; a Seção 6 expõe uma avaliação realizada sobre o JMBL concebido; Por fim, a Seção 7 apresenta a conclusão e os trabalhos futuros.

\section{Metodologia da Pesquisa}

Para o desenvolvimento deste artigo, inicialmente realizou-se uma pesquisa bibliográfica exploratória, que proporcionou uma visão geral sobre JMBLs, com objetivo de conhecer os conceitos fundamentais e aplicações. A partir deste levantamento bibliográfico, identificou-se trabalhos que conceberam JMBLs voltados para auxílio no processo de ensino-aprendizagem.

Após a revisão bibliográfica, iniciou-se o processo de levantamento de requisitos, no qual identificou-se os requisitos funcionais e não funcionais do JMBL. Em seguida, iniciou-se o processo de escolha dos elementos de jogos e procedimentos para o desenvolvimento das mecânicas de jogos. Posteriormente, realizou-se a modelagem das interfaces, e, por fim, a implementação e avaliação de caráter ergonômico (usabilidade) e de caráter pedagógico (aprendizagem), no qual estudantes e professores interagiram com o JMBL, com objetivo de mensurar a simplicidade, a 
eficiência das interfaces e, porfim, a relevância dos aspectos pedagógicos presentes no JMBL.

\section{Jogos Móveis Baseados em Localização}

Os jogos digitais têm por objetivo oferecer aos jogadores uma atividade lúdica, na qual os participantes as realizam pelo prazer e adrenalina que eles proporcionam. Os JMBLs diferem dos demais pela característica de não aprisionar os jogadores nas telas dos consoles, pois as aventuras presentes nos mesmos acontecem no espaço urbano, no qual o jogador passa a experimentar um jogo que se relaciona com o mundo real (BENFORD et al. 2005). As ações dos participantes no espaço urbano interferem diretamente no ambiente virtual. Os JMBLs são uma subclasse dos jogos pervasivos. De acordo com (WALTHER, 2005) os jogos pervasivos têm como característica fundamental utilizar tanto o espaço virtual como o real para possibilitar suas atividades.

Segundo (LEMOS, 2010), os JMBLs são jogos que utilizam o espaço público como espaço de jogo, por meio de Serviços Baseados em Localização (LBS) e Tecnologias Baseadas em Localização (LBT) para ação e desenvolvimento das mecânicas de jogo, tais como: Sistema de Posição Global (GPS), Wi-Fi, 3G, 4G, GPRS, acelerômetro, bússola magnética, giroscópio e dispositivos móveis. O uso desses dispositivos é que diferenciam os JMBLs de qualquer outro tipo de jogo pervasivo. A tecnologia de RA pode ser introduzida aos JMBLs com finalidade de fortalecer a realização das atividades lúdicas. A RA permite que as pessoas integrem objetos virtuais e transmitam dados diretamente no ambiente ao redor do usuário (DAPONTE et al. 2014).

\section{Trabalhos Relacionados}

Com o objetivo de identificar JMBLs que focam no processo de aprendizagem, realizou-se uma pesquisa bibliográfica exploratória sobre estudos que conceberam tais ferramentas de ensino. Dentre os estudos encontrados, (PRITAMI e MUHIMMAH, 2018) conceberam um JMBL que utiliza recursos de RA para apoiar o processo de ensino-aprendizagem sobre Matemática. Seu foco é estimular a capacidade de contagem da criança por meio de uma atividade lúdica. $\mathrm{O}$ jogo projeta no ambiente real objetos 3D em formato de balões com cores e localização distinta, em que os jogadores devem atirar em balões no qual encontram-se as respostas corretas para as perguntas fornecidas pelo sistema.

Em (VASSILAKIS et al. 2017), é concebido um JMBL cujo objetivo é ensinar a história das muralhas venezianas da cidade de Heraklion, no qual permite aos jogadores explorá-las de forma lúdica. O jogo fornece instruções de como chegar a um local específico, onde o jogador deve escanear a área de interesse, por meio da câmera de um dispositivo móvel, para que seja renderizado um objeto 3D no plano do jogo. Em seguida, é fornecida uma pergunta de múltipla escolha sobre o local escaneado, na qual o jogador deve tentar respondê-la de forma correta.

Apesar dos trabalhos relacionados fornecerem um espaço lúdico para os estudantes, estes não apresentam um ambiente que facilite a criação e o gerenciamento das atividades lúdicas disponíveis no JMBL. Por esta razão, além da aplicação móvel, 
apresentamos um ambiente web que permite aos professores adicionar sua didática ao jogo de forma fácil e intuitiva. O JMBL concebido também permite ao professor acessar o desempenho do estudante durante a interação com o jogo, verificando, assim, as deficiências de cada um deles. Além dos benefícios apresentados, o JMBL possibilita aos estudantes realizar a inclusão de conteúdos adicional ao jogo, o que permite que colaborem no processo de ensino-aprendizagem.

\section{JMBL Concebido}

A arquitetura do JMBL desenvolvido é composta por três componentes: i) aplicação móvel, ii) aplicação web e iii) servidor. Os componentes que integram o JMBL são estruturados com base no modelo cliente-servidor, no qual o servidor é o elemento central, cuja função é fornecer recursos para os demais.

\subsection{Aplicação Móvel}

A aplicação móvel é o ambiente no qual as atividades lúdicas são disponibilizadas aos estudantes. Desenvolvida para o sistema operacional Android, compatível a partir da versão 4.4, suas interfaces são projetadas para operar de forma responsiva, capaz de adaptar-se a diferentes tamanhos de telas. Para participar das atividades lúdicas, o estudante deve primeiramente criar uma conta na tela de cadastro. Após o cadastro, o estudante será direcionado a tela principal, composta por um mapa no qual encontram-se os conteúdos locativos (Figura 1(a)), são eles: desafios, provas e dicas.

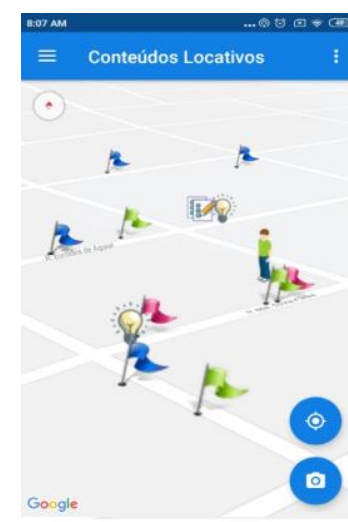

(a)

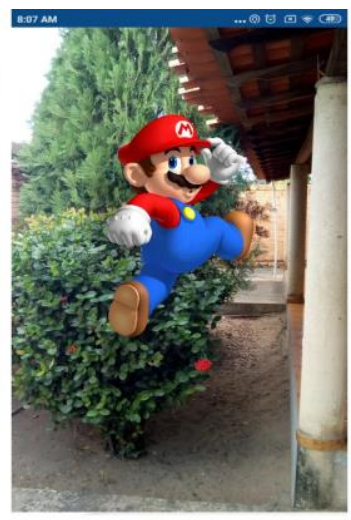

(b)

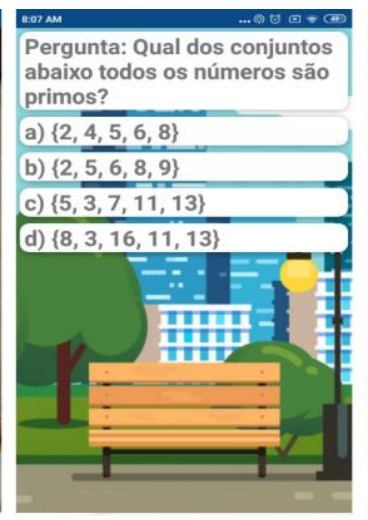

(c)

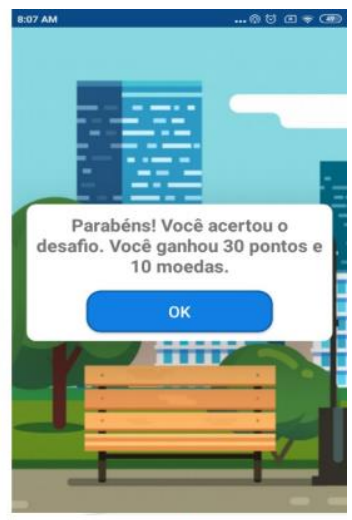

(d)

Figura 1 - Atividade lúdica executada pelo estudante.

Os desafios são quizzes locativos criados por professores e estudantes. A ideia de possibilitar aos estudantes criar desafios é proporcioná-los diferentes papéis no processo de aprendizagem. Os desafios concebidos pelos estudantes são enviados para professores e acessados pelos mesmos por meio da aplicação web. Ao aprová-los, os estudantes são recompensados com 2 moedas e os desafios são adicionados ao espaço urbano. As provas são compostas por um conjunto de questões objetivas de uma determinada área de conhecimento. Em que para concluí-la, o estudante deve responder corretamente todas as questões. Já as dicas ajudam os estudantes a concluírem desafios 
ou provas. Para adquirir estas dicas os estudantes devem comprá-las com moedas conquistadas ao longo do jogo. As provas e as dicas são criadas apenas por professores, por meio da aplicação web.

As atividades lúdicas do JMBL são realizadas da seguinte maneira. Primeiramente o estudante deve deslocar-se no espaço urbano até um determinado ponto onde encontra-se um conteúdo locativo, identifique-o e capture o objeto de RA (Figura 1(b)). Posteriormente é apresentado o conteúdo vinculado ao objeto capturado (Figura 1(c)). Caso o conteúdo seja de um desafio ou de uma prova, o estudante é convidado a respondê-lo. Ao conclui-lo, é apresentado uma mensagem referente à recompensa conquistada (Figura 1(d)). Em seguida, o objeto de RA é salvo na coleção. Caso o estudante não consiga concluir à atividade didática, é oferecida uma dica bônus e uma nova chance, com intuito de tornar o erro parte natural do processo de aprendizagem. Se o objeto de RA capturado for de uma dica, o estudante é convidado a comprá-la. Ao comprá-la, a dica é apresentada ao estudante.

A aplicação móvel possui um menu lateral (Figura 2(a)) acessado por meio de um ícone posicionado no canto superior esquerdo da tela. Por meio deste menu, 0 estudante tem acesso às demais funcionalidades da aplicação. A Figura 2(b) apresenta a tela do perfil do estudante, no qual é possível visualizar informações resultantes de suas ações. $\mathrm{Na}$ tela de perfil são apresentados os emblemas, no qual cada emblema representa um nível concluído. Para avançar de nível, além da pontuação necessária, ao final de um nível é preciso responder uma prova de conhecimentos gerais, de acordo com o nível do estudante, gerada a partir de desafios já cadastrados no banco de dados, adaptando assim, as tarefas aos níveis de habilidades dos estudantes.

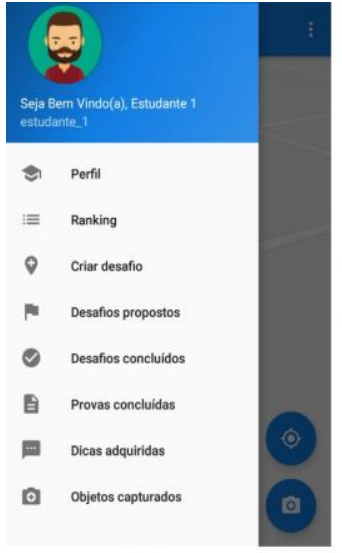

(a)

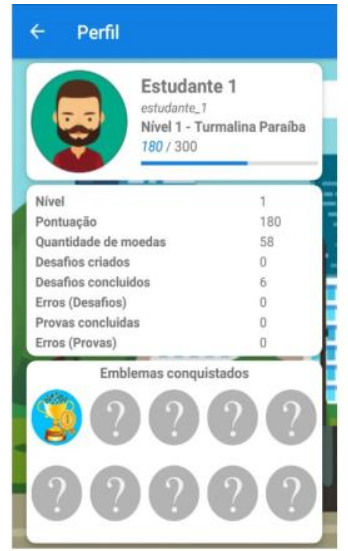

(b)

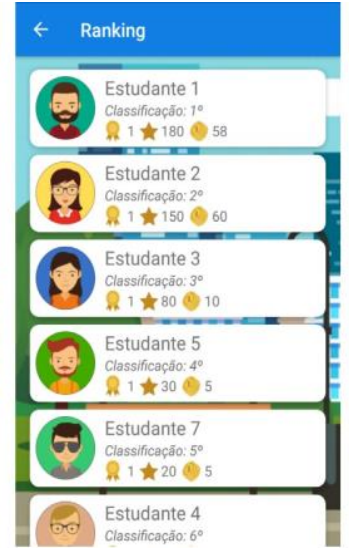

(c)

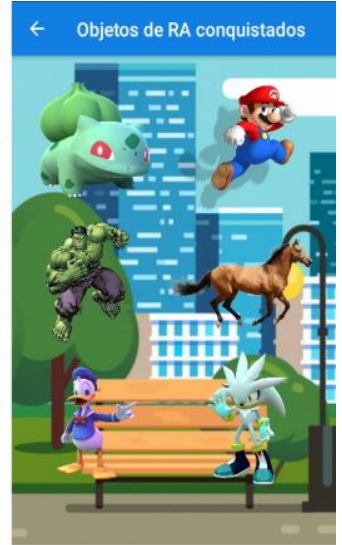

(d)

Figura 2 - (a) Menu (b) Perfil do estudante. (c) Ranking. (d) Objetos de RA capturados.

Os níveis de dificuldades da aplicação foram criados a partir de nomes de pedras preciosas, são elas: Turmalina Paraíba, Diamante, Rubi, Jadeíta, Musgravite, Painite, Grandidierite, Garnet Azul, Serendibite e Diamante Vermelho. Escolheu-se a ordem de cada pedra preciosa, com relação a ordem dos níveis, com base no seu valor de mercado. Portanto, foi definido o nível Turmalina Paraíba como nível inicial, e o Diamante Vermelho como nível máximo. A aplicação dispõe ainda de uma tela ranking que apresenta os estudantes ordenados em ordem decrescente de pontuação (Figura 
2(c)). Ao lado, na Figura 2(d) temos a tela de coleções, no qual é possível visualizar os objetos de RA conquistados durante as aventuras do jogo.

\subsection{Aplicação Web}

A aplicação web possibilita professores criar e gerenciar os conteúdos locativos acessados pelos estudantes e acompanhar o desempenho dos mesmos. Primeiramente, o professor deve realizar seu cadastro para usufruir das funcionalidades oferecidas pela aplicação, são elas: criar e gerenciar desafios, provas, dicas, e acompanhar o desempenho dos estudantes dentro do jogo. Após o cadastro, o professor é direcionado para tela principal, composta por um mapa no qual encontram-se todos os conteúdos locativos adicionados pelos demais professores e um menu com as principais funcionalidades da aplicação.Ao escolher a opção desafios no menu principal, o professor é direcionado para uma tela composta por um mapa e uma lista com todos os desafios já criados por ele (Figura 4).

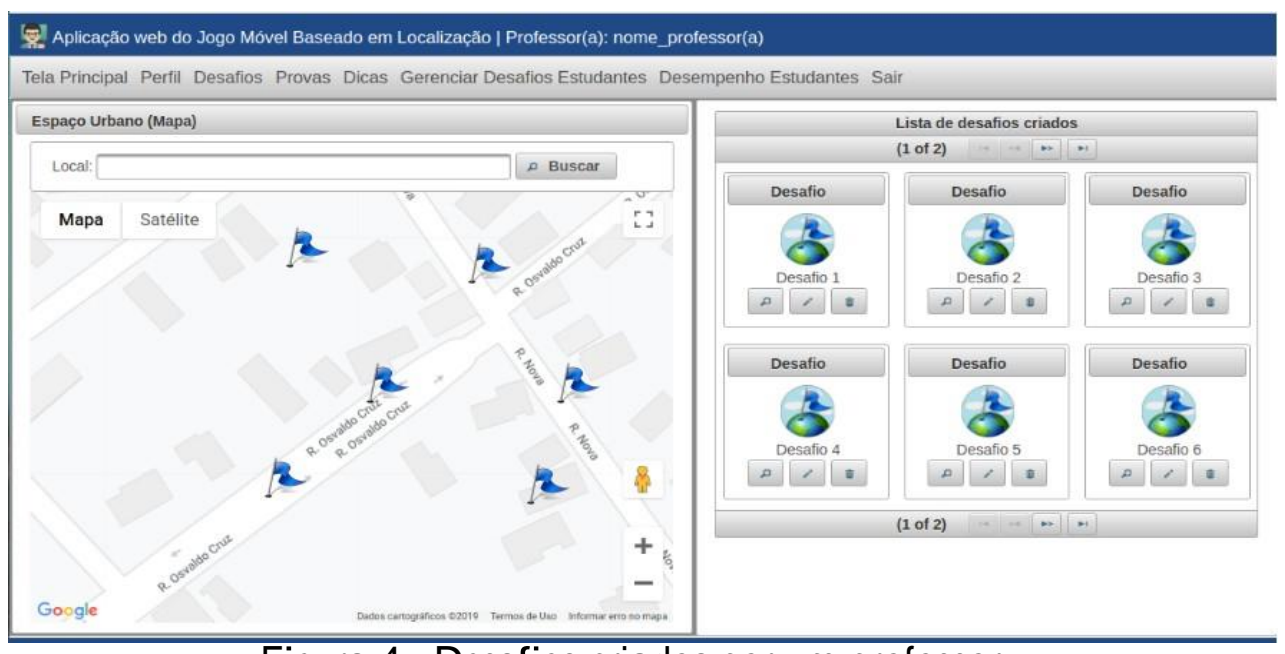

Figura 4 - Desafios criados por um professor.

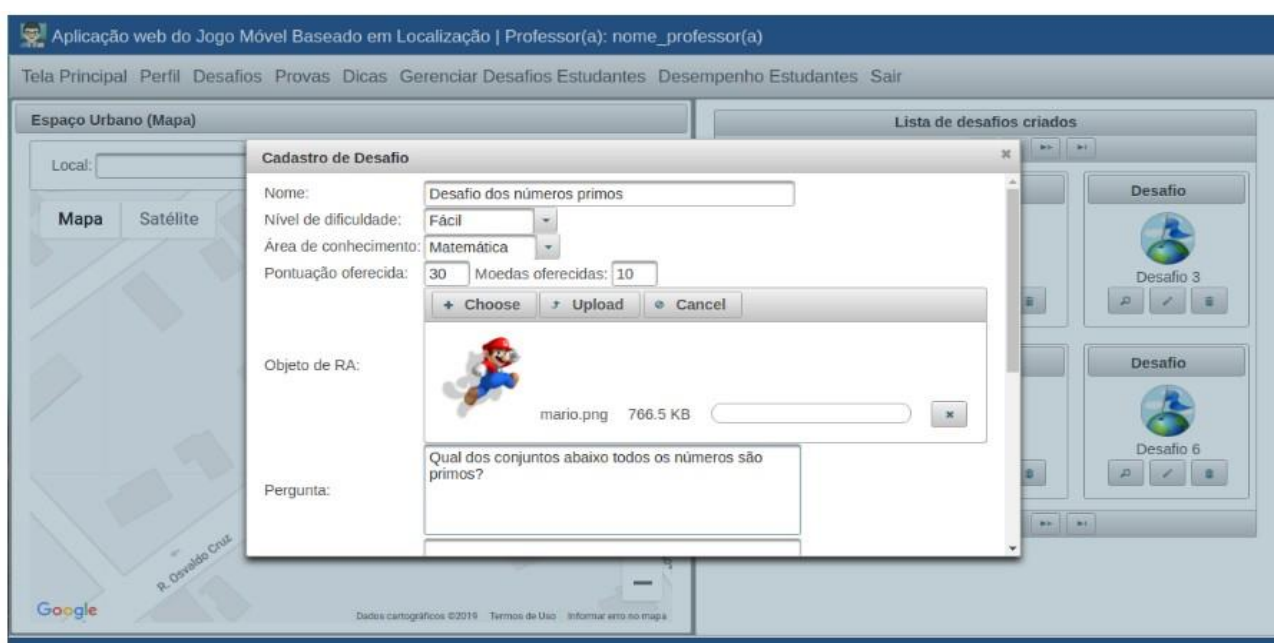

Figura 5: Cadastro de desafio. 
Para adicionar um desafio ao espaço urbano, o professor deve clicar em um local no mapa onde deseja adicioná-lo. A partir desta ação, é feita a navegação para a tela de criação do desafio (Figura 5), no qual são inseridas informações necessárias para a criação do desafio. $O$ mesmo processo é realizado para a criação das provas e dicas. A aplicação web possibilita ainda acompanhar o desempenho dos estudantes que participam das atividades lúdicas, apresentando resultados de suas ações em forma de gráficos e tabelas. Este acompanhamento tem como função descobrir as reais dificuldades e necessidades dos estudantes.

\section{Avaliação}

Nesta seção são descritos os aspectos avaliados e os procedimentos utilizados para avaliação do JMBL, o perfil dos avaliadores e os resultados obtidos após a execução do processo de avaliação.

\subsection{Procedimentos de Avaliação}

Em (NUNES e SANTOS, 2018) os autores apontam que muitos dos trabalhos que propõe softwares educativos, levam em consideração apenas normas técnicas de avaliação do processo e produto, e aspectos de usabilidade, e não observam a sua estrutura pedagógica. Com base neste contexto, realizou-se no presente artigo uma avaliação de caráter ergonômico-pedagógica, com intuito de mensurar a simplicidade e eficiência das interfaces do JMBL proposto, e aferir se o mesmo tem o potencial de contribuir de forma significativa para o processo de aprendizagem do estudante.

Para avaliação ergonômica considerou-se os seguintes aspectos apresentados por [NUNES e SANTOS 2018] para avaliar a usabilidade de aplicativos móveis educativos, tais como: controle do usuário (E1), saídas claramente demarcadas (E2), flexibilidade e eficiência de uso (E3), ajuda e documentação (E4), design estético e minimalista (E5), diálogos simples e naturais (E6) e mensagem de erro (E7). Considerou-se também a mesura do engajamento dos elementos de jogos presente no JMBL. Na avaliação pedagógica adaptou-se critérios elaborados por (VIEIRA et al. 2012) com objetivo de avaliar aspectos pedagógicos presente em softwares educativos, são eles: harmonia entre as atividades e os objetivos (P1), clareza e interatividade (P2), facilidade dos procedimentos (P3), aspectos motivadores (P4), oportunidade de retornar à atividade em caso de erro (P5), atividades reflexivas (P6) e feedback (P7).

O processo de avaliação ocorreu em dois momentos: no primeiro, o JMBL foi apresentado para 53 alunos do ensino médio de uma escola pública com o auxílio de um professor. No qual utilizaram o JMBL por algumas horas. Em seguida, aplicou-se um questionário composto por 16 perguntas, no qual 8 delas eram referentes à experiência de uso e as demais sobre o perfil de cada estudante; o segundo momento consistiu em apresentar o JMBL para 10 professores, todos com formação pedagógica, no qual foram convidados a criarem algumas atividades lúdicas utilizando a aplicação web, e em seguida, responderam um questionário com 12 perguntas, no qual 7 tinham o propósito de avaliar os aspectos pedagógicos presentes no JMBL, e as demais sobre o perfil de cada professor. 


\subsection{Perfil dos Avaliadores}

Após a execução do processo de avaliação descrito anteriormente, foi possível definir o perfil dos estudantes e professores que avaliaram o JMBL. Entre os estudantes, $17 \%$ tinham idade de 12 a 14 anos e $83 \%$ de 15 a 17 anos, no qual $54,7 \%$ eram do sexo feminino e $45,3 \%$ do sexo masculino. Com relação à utilização de jogos móveis educativos, $18,9 \%$ destes estudantes responderam que costumam utilizá-los com frequência, e 60,4\% responderam que costumam utilizá-los às vezes. Sobre os JMBLs, $88,7 \%$ dos estudantes disseram conhecer algum JMBL, mas apenas $39,6 \%$ já utilizou algum. Dentro do conjunto de professores, 40\% tinham idade de 31 a 40 anos, $50 \%$ de 41 a 50 anos e $10 \%$ acima de 50 anos, no qual $40 \%$ eram do sexo feminino e $60 \%$ do sexo masculino. Com relação à experiência na docência, 20\% tinham de 8 a 10 anos de experiência e $80 \%$ mais de 10 anos.

\subsection{Resultados Obtidos}

Sobre os aspectos ergonômicos avaliados, ao observar os dados do gráfico da Figura 6(a) pode-se perceber que o JMBL atende de forma satisfatória aos aspectos ergonômicos abordados. Com relação ao aspecto de controle do usuário (E1), o JMBL possibilita ao estudante o controle do encadeamento e a realização das ações, deixandoo que escolha as ações que serão executadas e como serão executadas. Quanto ao controle do sistema (E2), o estudante pode a qualquer momento desfazer ou abortar uma operação, retornando assim ao estado anterior.

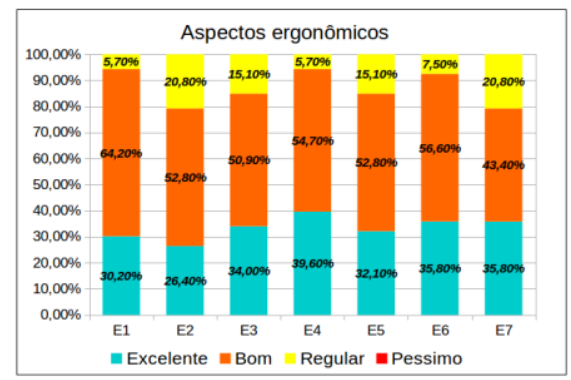

(a)

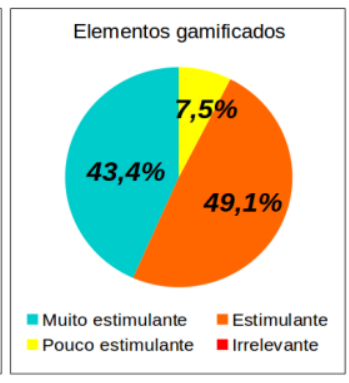

(b)

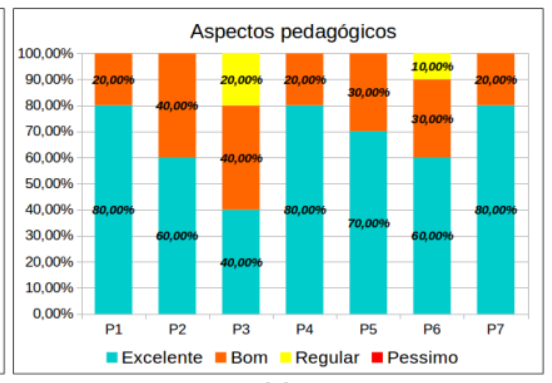

(c)

Figura 6 - (a) Resultados dos aspectos ergonômicos avaliados. (b) Resultado sobre 0 potencial dos elementos de jogos. (c) Resultados dos aspectos pedagógicos avaliados.

Quando questionados sobre a flexibilidade e eficiência de uso (E3), os estudantes responderam que o JMBL é familiar tanto para usuários avançados quanto para usuários leigos, no qual apresenta apenas as informações necessárias para aquele momento (E4). Mas caso o estudante venha a apresentar dificuldades em realizar determinados procedimentos na aplicação, a ajuda e documentação oferecida pelo JMBL (E5) é de fácil acesso e está sempre disponível.

No que se refere aos diálogos (E6), os estudantes definiram como simples, diretos e naturais. No que diz respeito às mensagens de erros (E7) são apresentadas de forma natural, com redação simples e clara, não o intimidando. Sobre o potencial dos elementos de jogos em engajar e motivar os estudantes na realização de atividades didáticas, notou-se por meio dos resultados apresentados no gráfico da Figura 6(b) que 
$92,5 \%$ dos estudantes, no qual $43,4 \%$ classificaram como muito estimulante e $49,1 \%$ como estimulante, sentiram-se motivados em participar das atividades por causa dos elementos de jogos inseridos no JMBL.

Quanto aos resultados dos aspectos pedagógicos avaliados, podemos afirmar diante dos resultados apresentados no gráfico da Figura 6(c), que o JMBL trabalha com uma estrutura pedagógica capaz de contribuir significativamente para o aprendizado do estudante. Sobre a qualidade do material pedagógico das atividades propostas (P1), $75 \%$ dos professores classificaram como excelente. De acordo com os professores, o JMBL orienta o estudante passo a passo nos procedimentos a serem seguidos (P2). Logo, o JMBL apresenta os processos de maneira a minimizar confusões, duvidas, insatisfação ou frustração do estudante (P3).

Com relação aos aspectos motivadores da aplicação (P4), os professores responderam que a aplicação tem o potencial de estimular e engajar o estudante durante 0 aprendizado. Além da motivação, o JMBL trata o erro como parte natural do processo de aprendizagem, possibilitando ao estudante retornar à atividade respondida de forma errada anteriormente (P5). Possibilita também a reflexão e interpretação das atividades oferecidas aos estudantes (P6). Implementou-se ainda um sistema de feedback eficiente (P7), no qual a cada ação do estudante dentro do jogo, é apresentado ao mesmo o resultado obtido por meio daquela ação executada, possibilitando ao estudante acompanhar e avaliar seu desempenho.

\section{Conclusão e Trabalhos Futuros}

Esse artigo concebeu um JMBL educativo com recursos de RA, que fornece aos estudantes um ambiente lúdico e engajador para motivá-los na realização de atividades escolares. O JMBL dispõe de uma aplicação móvel e uma aplicação web. A aplicação móvel foi desenvolvida para o sistema operacional Android, é o ambiente no qual são disponibilizadas as atividades lúdicas aos estudantes. Já a aplicação web possibilita professores criar e gerenciar o conteúdo didático acessado pelos estudantes por meio da aplicação móvel, e acompanhar o desempenho dos mesmos.

A análise dos resultados obtidos apontaram que o JMBL atende de forma satisfatória aos aspectos ergonômicos e pedagógicos avaliados. Portanto, concluiu-se que o JMBL concebido proporciona ao estudante uma experiência de uso agradável, motivadora e engajadora, e possui uma estrutura pedagógica com o potencial de colaborar no processo de ensino-aprendizagem do estudante. Como trabalhos futuros propõem-se a integração de objetos animados em 3D e a utilização de recursos multimídias como áudio e vídeo. Pretende-se também realizar uma avaliação pedagógica com um maior número de professores com formação pedagógica.

\section{Referências Bibliográficas}

BENFORD, S.; MAGERKURTH, C.; LJUNGSTRAND, P. Bridging the physical and digital in pervasive gaming. Communications of the ACM, v. 48, n. 3, p. 54-57, 2005. 
DAPONTE, $P$ et al. State of the art and future developments of the Augmented Reality for measurement applications. Measurement, v. 57, p. 53-70, 2014.

FERNANDES, C. W. R.; RIBEIRO, E. L. P. GAMES, GAMIFICAÇÃO E O CENÁRIO EDUCACIONAL BRASILEIRO. CIET: EnPED, 2018.

KAPP, Karl. The Gamification of Learning and Instruction: Game-based Methods and Strategies for Training and Education. Pfeiffer, 2012.

LEMOS, A. Jogos móveis locativos: Cibercultura, espaço urbano e mídia locativa. Revista USP, São Paulo. n.86, p.54-65, junho/agosto. 2010.

NUNES, R. P.; SANTOS, I. M. A importância da avaliação ergonômico-pedagógica de aplicativos educacionais e os desafios encontrados no campo da aprendizagem móvel. In: Simpósio Brasileiro De Informática Na Educação. 2018. p 41.

PRITAMI, F. A.; MUHIMMAH, I. Digital game based learning using augmented reality for mathematics learning. In: Proceedings of the 2018 7th International Conference on Software and Computer Applications. ACM, 2018. p. 254-258.

UZUNCA, B.; JANSEN, S. How do Ecosystem Dynamics work in Serious Gaming Ecosystems? Challenges and Opportunities. Strategic Management Society, 2016.

Vassilakis, K., Charalampakos, O., Glykokokalos, G., Kontokalou, P., Kalogiannakis, M., e Vidakis, N. Learning history through location-based games: The fortification gates of the venetian walls of the city of Heraklion. In: Interactivity, Game Creation, Design, Learning, and Innovation. Springer, Cham, 2017. p. 510-519.

VIEIRA, M. M. S.; SIMÕES, L. L. F.; BARRETO, A. L. de O. Avaliação De Software Educativo: Aspectos Pedagógicos E Técnicos. Revista Faculdade Cearense (FAC), Fortaleza, v. 5 nํ..1, 2012.

WALTHER, B. K. (2005). Reflections on the methodology of pervasive gaming. In: Proceedings of the 2005 ACM SIGCHI International Conference on Advances in computer entertainment technology. ACM, 2005. p. 176-179. 